\title{
Applying 3DPCANet and Functional Magnetic Resonance Imaging to Aided Diagnosis of Alzheimer's Disease
}

Yu Wang ( $\nabla$ wangyu@btbu.edu.cn )

Beijing Technology and Business University

Hongfei Jia

Beijing Technology and Business University

Yifan Duan

Beijing Technology and Business University

Hongbing Xiao

Beijing Technology and Business University

\section{Research Article}

Keywords: Alzheimer's disease, functional magnetic resonance imaging, 3DPCANet, support vector machine, mean amplitude of low-frequency fluctuation

Posted Date: August 26th, 2021

DOl: https://doi.org/10.21203/rs.3.rs-820356/v1

License: (9) This work is licensed under a Creative Commons Attribution 4.0 International License. Read Full License 


\section{Applying 3DPCANet and Functional Magnetic Resonance \\ 2 Imaging to Aided Diagnosis of Alzheimer's Disease}

3 Yu Wang ${ }^{1}$, Hongfei Jia ${ }^{1}$, Yifan Duan ${ }^{1}$, Hongbing Xiao ${ }^{1}$

41 Beijing Key Laboratory of Big Data Technology for Food Safety, School of Artificial

5 Intelligence, Beijing Technology and Business University, Beijing 100048, China.

6 Correspondence should be addressed to Yu Wang: wangyu @btbu.edu.cn; Hongfei Jia: jiaho

7 ngfei1947@163.com; Yifan Duan: 1106811434@qq.com; Hongbing Xiao: x.hb@163.com

8 Abstract

9 Alzheimer's disease (AD) is a progressive neurodegenerative disease, which changes the 10 structure of brain regions by some hidden causes. In this paper for assisting doctors to make 11 correct judgments, an improved 3DPCANet method is proposed to classify AD by combining 12 the mean (mALFF) of the whole brain. The main idea includes that firstly, the functional 13 magnetic resonance imaging (fMRI) data is pre-processed, and mALFF is calculated to get the corresponding matrix. Then the features of mALFF images are extracted via the improved 3DPCANet network. Finally, AD patients with different stages are classified using support vector machine (SVM). Experiments results based on public data from the Alzheimer's disease neuroimaging initiative (ADNI) show that the proposed approach has better performance compared with state-of-the-art methods. The accuracies of AD vs. significant memory concern (SMC), SMC vs. late mild cognitive impairment (LMCI), and normal control (NC) vs. SMC reach respectively $92.42 \%, 91.80 \%$, and $89.50 \%$, which testifies the feasibility and effectiveness of the proposed method.

Keywords: Alzheimer's disease; functional magnetic resonance imaging; 3DPCANet; support vector machine; mean amplitude of low-frequency fluctuation

0 Introduction

Alzheimer's disease (Alzheimer's disease, AD) is one of the most common neurological diseases of the brain, which seriously endangers the life and health of patients. By the end of 2019, more than 10 million AD patients occurred in China which had the largest number of people with $\mathrm{AD}$ in the world [1]. AD firstly attacks the hippocampus where study and memory are processed [2]. In the early stage of this disease, the symptoms are forgetfulness and confusion. With the aggravation of the disease, visual space of the patient decreases, and patients cannot distinguish or deal with things alone. Patients' memories loss is serious, which leads to heavy burden for both patients and society. Therefore, the early diagnosis and timely treatment of $\mathrm{AD}$ is of great significance. In recent years, with the rapid development of computer and neuroimaging, it has become a mainstream trend to use computers and medical imaging to diagnose and analyse AD. Jiang [3] et al. VGGnet is used to extract normal control (NC) and early mild cognitive impairment (EMCI) sMRI image the deep 
embedded features of the image, and Lasso is used to select the extracted features. Finally, SVM is used to classify the selected features. At present, the technical difficulties mainly include the extraction of effective features in medical imaging, as well as the design and construction of classification models with good robustness and simple structure.

Functional magnetic resonance imaging (fMRI) [4-5] is a non-invasive method which measures blood oxygen level dependent (BOLD) signal in the brain region at a certain moment and are widely used in AD research [6-9]. Zhou [10] et al. analyzed the locations of the lesion area, and used kernel principal component analysis (KPCA) to extract features. Finally, Adaboost is used to classify AD. Based on the automatic anatomical marker template, $\mathrm{Li}$ [11] et al. divided brain fMRI of each AD subject into 116 brain regions, and constructed a full brain function connection matrix by extracting the time series of each brain region. Then KPCA is used for feature extraction, and adaboost algorithm is used to classify. Using traditional machine learning methods like KPCA nonlinear image features can be extracted, but deep features are lacking. Therefore, in this paper, the traditional 3DPCANet network is improved. Max-pooling layer and activation function layer are added behind the convolutional layer for increasing the depth of network. So, the deep and nonlinear image features are extracted, and the classification accuracy of AD patients is increased.

Convolutional Neural Networks (CNN) is a feedforward neural network which is one of classic deep learning algorithms, and is mainly used for image feature extraction and classification. More and more researchers combine $\mathrm{CNN}$ with medical imaging to assist doctors for diagnosing diseases [12-15]. Rachna [16] et al. proposed a CNN model based on the transfer learning, in which the ImageNet dataset was used to pre-train VGG-16, and patients with the AD, NC, and MCI were then classified. Zhang [17] et al. designed a CNN using positron emission computed tomography (PET) and magnetic resonance imaging (MRI) as input. The obtained features were fused with those obtained by the mini-mental state examination (MMSE) and the clinical dementia rating (CDR). Then the fused features were inputted into $\mathrm{CNN}$ to realize the classification of $\mathrm{AD}, \mathrm{NC}$ and MCI. Most of the research content of the above papers is to classify $\mathrm{AD}, \mathrm{NC}$ and $\mathrm{MCI}$, which does not accurately diagnose the stage of the patient with AD. Significant memory concern (SMC) stage exists between NC and MCI, and MCI patients are divided into EMCI and late mild cognitive impairment (Late MCI, LMCI). Compared with EMCI, patients with LMCI showed significant declines in cognition, memory, and self-care. Due to the small changes in the brain structure of $\mathrm{AD}$ patients on adjacent development stages, it is difficult to extract effective classification features. While the model proposed in this paper may be used to classify more detailed subgroups of $\mathrm{AD}$ patients, and to better assist medical personnel to diagnose the disease.

PCANet is a simple CNN, proposed by Chan [18] et al, by which the deep features of the sample data are learned based on the traditional principal component analysis (PCA). Li [19] developed 3D-PCANet deep network for MRI feature extraction, and realizes the automatic classification of MRI by combining the obtained data information with the support vector machine (SVM) classifier. Although 3DPCANet can extract the deep features of the image, the phenomenon of feature redundancy will happen which gravely affects the image classification. For solving this problem, the traditional 3DPCANet is improved in this paper. The max-pooling layer is added behind each layer of convolutional layer. Therefore, the redundant features are reduced. In addition, the activation function layer (rectified linear unit, ReLU) is added for learning nonlinear features. Because the overall depth of the proposed 
model is deepened, deeper features of the images can be extracted. Although CNN combining with medical images has shown excellent performance in AD-assisted diagnosis, most of CNNs can only process two-dimensional images and cannot deal with high-dimensional data, especially four-dimensional fMRI data. Therefore, in this paper four-dimensional fMRI data is firstly changed into three-dimensional data. Then 3DPCANet model improved by adding max-pooling layer is used to extract features of fMRI data. Finally, SVM is used for classification. The experimental results verified the effectiveness of the proposed method. Contributions of this paper are as follows.

(1) The fMRI scan image is four dimensional, features cannot be directly extracted. In the experiment, fMRI images are converted to mean amplitude of low-frequency (mALFF) 3D image for feature extraction;

(2) Replace the manual function extraction with CNN. As a result, human error can be reduced and more desirable classification characteristics can be extracted.

(3) We can improve the 3DPCANet network and reduce the redundancy of functions by adding a maximum pooling layer after each layer of the line layer.

(4) Classify AD patients at different stages in order for medical personnel to accurately diagnose them.

\section{Materials and Methods}

\subsection{Experimental data preprocessing}

The fMRI data used in this experiment came from Alzheimer's Disease Neuroimaging Initiative (ADNI). The experimental data contained fMRI image data of 202 subjects, including $34 \mathrm{AD}$ patients, $57 \mathrm{EMCI}$ patients, $35 \mathrm{LMCI}$ patients, $50 \mathrm{NC}$ control groups, and $26 \mathrm{SMC}$ patients. The basic information for each subject in the experimental data is shown in Table 1.

Table 1: Statistical analysis of subjects' information.

\begin{tabular}{cccc}
\hline fMRI & Number of people & Male/Female & Age \\
\hline AD & 34 & $18 / 16$ & $57 \sim 88$ \\
EMCI & 57 & $34 / 23$ & $57 \sim 90$ \\
LMCI & 35 & $14 / 21$ & $58 \sim 88$ \\
NC & 50 & $28 / 22$ & $66 \sim 91$ \\
SMC & 26 & $14 / 12$ & $65 \sim 83$ \\
\hline
\end{tabular}

The pre-processing of fMRI data is implemented based on data processing \& analysis for brain imaging (DPABI) [20] software package. The fMRI image processing includes eliminating the first 10 time points, time horizon correction, head movement correction, spatial normalization, nonlinear drift and other processing. The fMRI pre-processed sample data is shown in Figure 1.
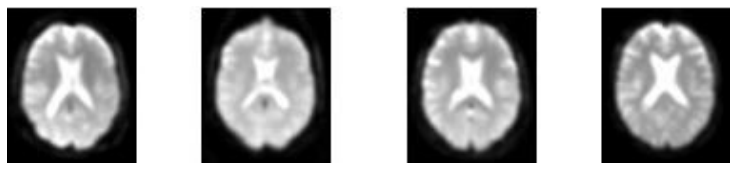

Figure 1: Sample images after fMRI pre-treatment 
116 fMRI data is 4-dimensional data. At present, few CNNs exist for feature extraction of high-

117 dimensional data, and most models are suitable for two-dimensional or three-dimensional

118 images. In this paper, the fMRI data is converted into mALFF [21] image which is a form of

$1193 \mathrm{D}$ image and is convenient to feature extraction using CNN. The ALFF image value is the

120 square root of the power spectrum of the BOLD signal in the low frequency band

$121(0.01 \mathrm{~Hz} \sim 0.08 \mathrm{~Hz})$. Low-frequency signal energy can be used to express the activity of

122 neurons in different brain regions. The activity of cerebral cortex neurons can be reflected by

123 the signal energy with frequency from $0.010 \mathrm{~Hz}$ to $0.027 \mathrm{~Hz}$, and the activity of basal ganglia

124 can be reflected by that with frequency from $0.027 \mathrm{~Hz}$ to $0.073 \mathrm{~Hz}$. These areas will be

125 influenced by AD. So ALFF is selected for image transformation. The ALFF calculation

126 process is as follows:

127 (1) The time series of each voxel after removing the linear drift are first passed through a

$128 \quad 0.01 \mathrm{HZ}-0.08 \mathrm{HZ}$ band pass filter.

129 (2) The filtering result is subjected to fast fourier transform, and the power spectrum is 130 obtained.

131 (3) The average value of the square root of power spectrum within $0.01-0.08 \mathrm{~Hz}$ is calculated, 132 which is ALFF.

133 Mean amplitude of low frequency fluctuations (mALFF) is obtained by dividing the average 134 amplitude of low frequency fluctuations (ALFF) of all voxels in the whole brain. Because the 135 brain structure of AD patients has changed, it is believed that the activity of neurons in each 136 brain area will also change compared with the normal control group. The pre-processed fMRI 137 data is further calculated mALFF. And the image after mALFF transformation is shown in 138 Figure 2.
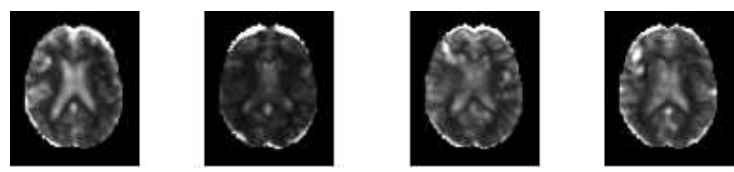

Figure 2: mALFF sample image

\section{$141 \quad 1.2$ Improvement of 3DPCANet model}

142 PCANet is a deep learning network in which uses PCA is used to learn channel convolution 143 kernels, and to made binary hashes and statistics of blocks histograms for indexing and 144 pooling. Li [19] et al. developed the PCANet model as a three-dimensional form for auxiliary 145 AD diagnosis. On this basis, in this paper the max-pooling layer is added to improve the 146 network behind the convolutional layer of the 3DPCANet model. The improved 3DPCANet convolutional neural network structure is shown in Figure 3. 


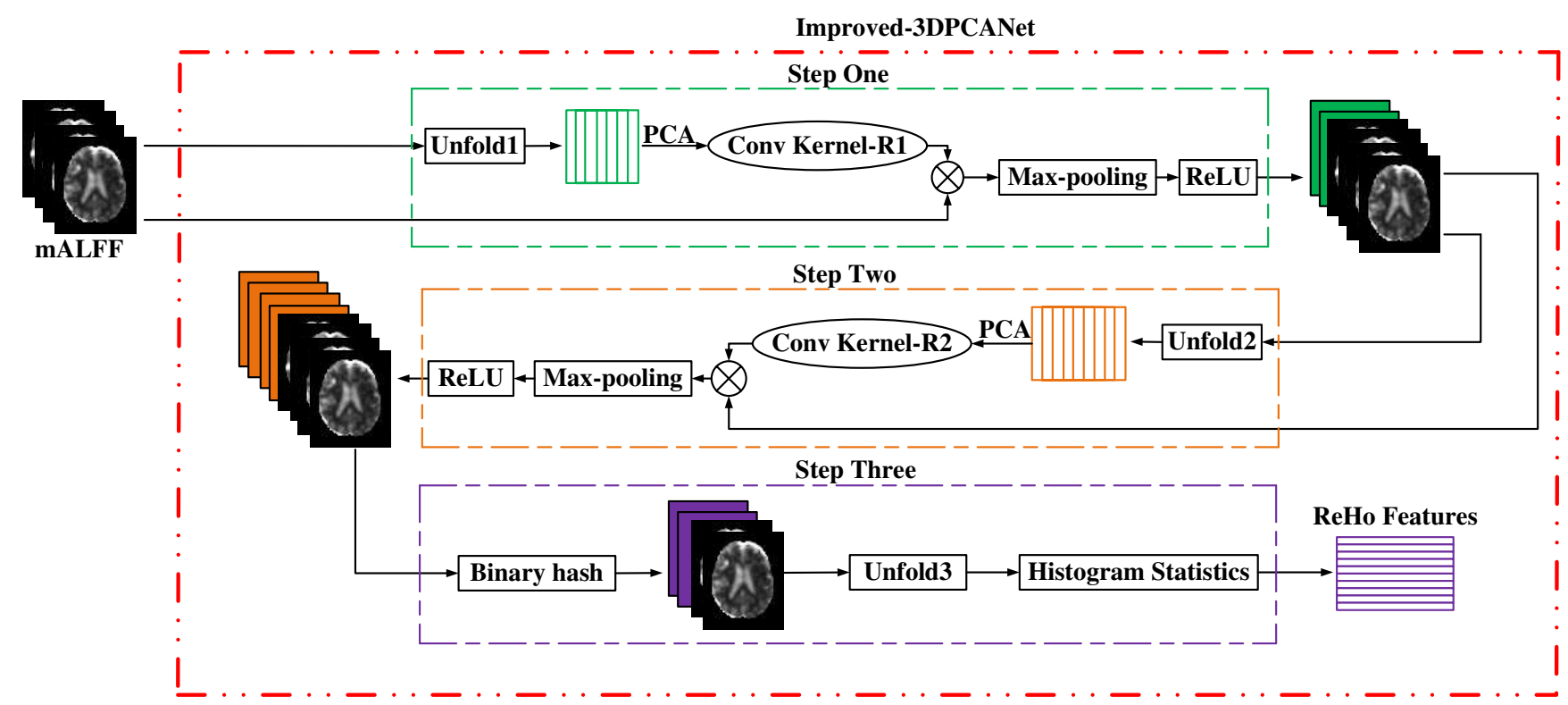

Figure 3: The framework of improved 3DPCANet.

$150 N$ training image $\left\{\boldsymbol{\Gamma}_{j}\right\}_{j=1}^{N}$ of size $L \times W \times H$ as the 3DPCANet input, 3DPCANet extracted 151 features process as follows.

152 (1) Input Layer

153 Voxel blocks with size $k_{1} \times k_{2} \times k_{3}$ around each voxel on the $j$-th training image is divided.

154 The total number of blocks intercepted in each image is $\beta_{1}$. i.e., $\boldsymbol{x}_{j, 1}, \boldsymbol{x}_{j, 2}, \ldots, \boldsymbol{x}_{j, i}, \ldots \boldsymbol{x}_{j, \beta_{1}}$, where 155 each $x_{j, i}$ represents the column vector of the $i$-th block in the $j$-th training image. These 156 blocks are taken out and reshaped into a column vector, and standardization processing is performed. As shown in the following formula.

$$
\overline{\boldsymbol{X}_{j}}=\left[\overline{\boldsymbol{x}_{j, 1}}, \overline{\boldsymbol{x}_{j, 2}}, \ldots, \overline{\boldsymbol{x}_{j, i}}, \ldots, \overline{\boldsymbol{x}_{j, \beta_{1}}}\right]
$$

Perform the above processing on all $N$ training images, and arrange the processed $N$ training images sequentially into a new matrix $X$ to obtain the following matrix.

$$
\boldsymbol{X}=\left[\overline{\boldsymbol{X}_{1}}, \overline{\boldsymbol{X}_{2}}, \ldots, \overline{\boldsymbol{X}_{j}}, \ldots, \overline{\boldsymbol{X}_{N}}\right]
$$

(2) Middle Layer

$T_{1}, T_{2}$ is filtering parameters of PCA in the two stages.

First stage PCA: For the matrix $\boldsymbol{X}$ is used PCA, PCA minimizes the reconstruction error on a group of standard orthogonal filters described as.

$$
\min _{\boldsymbol{V} \in \mathrm{i}}\left\|\boldsymbol{X}-\boldsymbol{V} \boldsymbol{V}^{\mathrm{T}} \boldsymbol{X}\right\|_{F}^{2} \text { s.t. } \boldsymbol{V}^{\mathrm{T}} \boldsymbol{V}=\boldsymbol{I}_{T_{1}}
$$

where $\boldsymbol{I}_{T_{1}}$ is the identity matrix with size $T_{1} \times T_{1}$. The solution $\boldsymbol{V}$ of this formula is the eigenvector of $\boldsymbol{X} \boldsymbol{X}^{\mathrm{T}}$. The expression of the PCA filter is as shown in equation (4).

$$
\boldsymbol{w}_{l}^{1}=\operatorname{mat}\left(\mathrm{q}_{l}\left(\boldsymbol{X} \boldsymbol{X}^{\mathrm{T}}\right)\right) \in \mathrm{i}, l=1,2, \ldots, T_{1}
$$

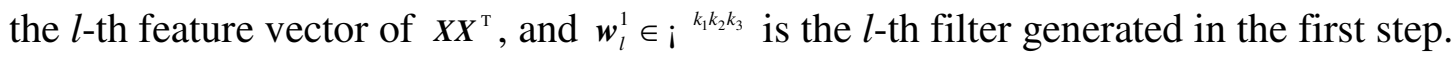


The PCA filter is convolved with the $j$-th training image $\Gamma_{j}$ in the original image, which is expressed by the formula (5).

$$
\boldsymbol{\Gamma}_{j, l}^{1}=\boldsymbol{\Gamma}_{j} * \boldsymbol{w}_{l}^{1}
$$

175

176

177

178

179

180

where the symbol ' $*$ ' represents convolution, and the filter $\mathrm{b}$ is used to convolve all $N$ training images and to generate $N * T_{1}$ images. Then the max-pooling layer and processing on non-linear activation function ReLU are performed on the image $\Gamma_{j, l}^{1}$ generated by formula (5), which is expressed by the following equation.

$$
\boldsymbol{\Pi}_{j, l}^{1}=\operatorname{ReLU}\left(\boldsymbol{\Gamma}_{j, l}^{1} \times \boldsymbol{P}^{1}\right)
$$

where the symbol ' $x$ ' represents the max-pooling operation. $\boldsymbol{P}^{1}$ denotes the max-pooling layer in the first step, and $\boldsymbol{\Pi}_{j, l}^{1}$ represents the image after the maximum pooling layer is processed.

Second stage PCA calculate: The $j$-th image in the $N$ training images by the first step generates $T_{1}$ images, among which $l$-th image performed operations similar equations (1) and (2) to get matrix $\boldsymbol{Y}$.

$$
\boldsymbol{Y}=\left[\overline{\boldsymbol{Y}_{1,1}}, \overline{\boldsymbol{Y}_{1,2}}, \ldots, \overline{\boldsymbol{Y}_{j, 1}}, \overline{\boldsymbol{Y}_{j, 2}}, \ldots, \overline{\boldsymbol{Y}_{j, l}}, \ldots, \overline{\boldsymbol{Y}_{N, 1}}, \overline{\boldsymbol{Y}_{N, 2}}, \ldots, \overline{\boldsymbol{Y}_{N, T_{1}}}\right]
$$

The filter $\boldsymbol{w}_{h}^{2}$ is obtained by the first stage PCA on the matrix $\boldsymbol{Y}$. The image $\boldsymbol{\Pi}_{j, l}^{1}$ generated by formula (6) in the first step is convolved by the obtained PCA filter, which is described by formula (8).

$$
\boldsymbol{\Pi}_{j, l, h}^{2}=\boldsymbol{\Pi}_{j, l}^{1} * \boldsymbol{w}_{h}^{2}
$$

Among $N * T_{1}$ images generated in the first step, each image is used to generate $T_{2}$ images by formula (8). The maximum pooling layer and non-linear activation function ReLU processing is performed on the image $\boldsymbol{\Pi}_{j, l, h}^{2}$ after convolution, as shown in the following formula.

$$
\boldsymbol{\Omega}_{j, l, h}^{2}=\operatorname{ReLU}\left(\boldsymbol{\Pi}_{j, l, h}^{2} \times \boldsymbol{P}^{2}\right)
$$

Where $\boldsymbol{P}^{2}$ denotes the max-pooling layer in the second step, and the image $\boldsymbol{\Omega}_{j, l, h}^{2}$ is generated by max-pooling operation.

(3) Output Layer

The heaviside function $\mathrm{H}(\mathrm{g})$ is used to binarize all $N * T_{1} * T_{2}$ images, and weighted processing to get $\boldsymbol{O}_{j, l}$.

$$
\boldsymbol{O}_{j, l}=\sum_{h=1}^{T_{2}} 2^{d-1}\left\{\mathrm{H}\left(\boldsymbol{\Omega}_{j, l, h}^{2}\right)\right\}
$$

Finally, $\beta$ blocks with size $k_{7} \times k_{8} \times k_{9}$ from $\boldsymbol{O}_{j, l}$ each image is divided. The histogram of each block is made statistics and vectorize the above results.

$$
\boldsymbol{F}_{j}=\left[\operatorname{Bhist}\left(\boldsymbol{O}_{j, 1}\right), \operatorname{Bhist}\left(\boldsymbol{O}_{j, 2}\right), \ldots, \operatorname{Bhist}\left(\boldsymbol{O}_{j, i}\right), \ldots, \operatorname{Bhist}\left(\boldsymbol{O}_{j, T_{1}}\right)\right]
$$

where $\operatorname{Bhist}\left(\boldsymbol{O}_{j, l}\right)$ is a function of block division, histogram statistics and concatenation of image $\boldsymbol{O}_{j, l} \cdot \boldsymbol{F}_{j}$ represents the final eigenvector of the $j$-th training images $\boldsymbol{\Gamma}_{j}$ using 3DPCANet 


\section{Results and Discussion}

211 In order to verify the robustness and effectiveness of the proposed model proposed in this

212 paper, a series of experiments for patients with different stages of AD and NC control group

213 were designed.

\section{$214 \quad 2.1$ Evaluation Criteria}

215 In the experiments, the data set is classified into two categories including positive and 216 negative samples. The evaluation criteria used in these experiments are accuracy, sensitivity 217 and specificity. The calculation equations are as follows:

$$
\begin{gathered}
\text { Accuracy }=\frac{T P+T N}{T P+F N+F P+T N} \\
\text { Sensitivity }=\frac{T P}{T P+F N} \\
\text { Specificity }=\frac{T N}{F P+T N}
\end{gathered}
$$

219 TP and TN respectively represent the number of true-positive subjects and true-negative subjects. FP and FN respectively represent the number of false-positive subjects and falsenegative subjects. The positive class label is 1 , and the negative class label is 0 . In order to comprehensively evaluate the proposed model, F1 score and the area under curve (AUC) are also used as evaluation indicators. The F1 value is calculated by precision and sensitivity, as shown in the formula (18).

$$
\begin{gathered}
\text { Precision }=\frac{T P}{T P+F P} \\
\mathrm{~F} 1=\frac{2 * \text { Precision } * \text { Sensitivity }}{(\text { Precision }+ \text { Sensitivity })}
\end{gathered}
$$

\subsection{Experimental results and analysis}

227 The experimental data included 34 AD patients, 57 EMCI patients, 35 LMCI patients, 26 228 SMC patients and 50 normal control groups. In order to better test the effectiveness of 229 mALFF images for AD classification and the robustness of the improved 3DPCANet model 230 in this paper, a series of experiments were carefully designed and 5-fold cross-validation was 231 used to reduce the contingency of experimental results.

232 The proposed method in this paper was compared with ones proposed in the literature [19] and the literature [22], [23], [24], [25], and the experimental results are shown in Table 2. In addition, AD patients at different stages also classified in this paper, and the experimental results were shown in Table 3 . 
Table 2: Comparison of experimental results.

\begin{tabular}{|c|c|c|c|c|c|}
\hline Model & indicators & NC vs. AD & SMC vs. LMCI & $\mathrm{NC}$ vs. EMCI & LMCI vs. AD \\
\hline \multirow{3}{*}{$\operatorname{Li}[19]$} & ACC & $83.95 \%$ & - & $80.15 \%$ & $82.53 \%$ \\
\hline & AUC & $88.42 \%$ & - & $81.74 \%$ & $81.24 \%$ \\
\hline & $\mathrm{ACC}$ & $78.95 \%$ & - & - & - \\
\hline \multirow[t]{2}{*}{ Dai [22] } & SEN & $81.25 \%$ & - & - & - \\
\hline & SPE & $77.27 \%$ & - & - & - \\
\hline \multirow{4}{*}{ Ashkan [23] } & ACC & $80.00 \%$ & - & $70.00 \%$ & $83.33 \%$ \\
\hline & SEN & $80.00 \%$ & - & $50.00 \%$ & $80.00 \%$ \\
\hline & $\mathrm{F} 1$ & $79.80 \%$ & - & $66.70 \%$ & $82.50 \%$ \\
\hline & $\mathrm{ACC}$ & $84.17 \%$ & - & $70.38 \%$ & $62.84 \%$ \\
\hline \multirow{3}{*}{ Beheshti [24] } & SEN & $88.83 \%$ & - & $78.17 \%$ & $76.38 \%$ \\
\hline & SPE & $79.00 \%$ & - & $60.22 \%$ & $39.57 \%$ \\
\hline & AUC & $86.00 \%$ & - & $72.00 \%$ & $64.00 \%$ \\
\hline \multirow[t]{2}{*}{ Korolev [25] } & $\mathrm{ACC}$ & $87.20 \%$ & - & $55.80 \%$ & $59.98 \%$ \\
\hline & AUC & $78.91 \%$ & - & $53.91 \%$ & $61.92 \%$ \\
\hline \multirow{6}{*}{ Ys [26] } & $\mathrm{ACC}$ & $87.30 \%$ & - & $70.50 \%$ & - \\
\hline & SEN & $91.40 \%$ & - & $75.2 \%$ & - \\
\hline & SPE & $79.30 \%$ & - & $63.8 \%$ & - \\
\hline & AUC & $89.10 \%$ & - & $78.00 \%$ & - \\
\hline & $\mathrm{ACC}$ & $87.78 \%$ & $91.80 \%$ & $81.82 \%$ & $86.67 \%$ \\
\hline & SEN & $96.00 \%$ & $91.43 \%$ & $80.00 \%$ & $88.57 \%$ \\
\hline 3DPCANet & SPE & $77.50 \%$ & $92.67 \%$ & $84.00 \%$ & $85.00 \%$ \\
\hline$+\mathrm{SVM}+\mathrm{mALFF}$ & $\mathrm{F} 1$ & $90.05 \%$ & $92.72 \%$ & $82.62 \%$ & $85.35 \%$ \\
\hline \multirow[t]{2}{*}{ (our) } & AUC & $88.50 \%$ & $88.09 \%$ & $78.17 \%$ & $83.93 \%$ \\
\hline & $\mathrm{ACC}$ & $88.89 \%$ & $91.80 \%$ & $87.27 \%$ & $89.33 \%$ \\
\hline Improved & SEN & $86.00 \%$ & $94.28 \%$ & $80.00 \%$ & $85.71 \%$ \\
\hline 3DPCANet+SVM & SPE & $92.50 \%$ & $88.67 \%$ & $96.00 \%$ & $92.50 \%$ \\
\hline \multirow[t]{2}{*}{ +mALFF (our) } & $\mathrm{F} 1$ & $89.57 \%$ & $92.92 \%$ & $87.21 \%$ & $88.08 \%$ \\
\hline & AUC & $82.25 \%$ & $90.86 \%$ & $\mathbf{8 9 . 8 3 \%}$ & $83.57 \%$ \\
\hline
\end{tabular}

Table 3 Classification of patients with different stages of AD

\begin{tabular}{cccccc}
\hline indicators & NC vs. SMC & SMC vs. EMCI & SMC vs. AD & EMCI vs. LMCI & EMCI vs. AD \\
\hline ACC & $89.50 \%$ & $88.43 \%$ & $92.42 \%$ & $84.21 \%$ & $88.42 \%$ \\
SEN & $94.00 \%$ & $91.67 \%$ & $88.00 \%$ & $88.33 \%$ & $90.00 \%$ \\
SPE & $80.67 \%$ & $81.33 \%$ & $95.00 \%$ & $77.14 \%$ & $85.71 \%$ \\
F1 & $90.10 \%$ & $91.65 \%$ & $89.46 \%$ & $87.44 \%$ & $90.79 \%$ \\
AUC & $89.53 \%$ & $79.55 \%$ & $86.83 \%$ & $80.95 \%$ & $85.48 \%$ \\
\hline
\end{tabular}

240 The conclusions can be drawn from the experimental results in Table 2. First, the 241 3DPCANet+SVM+mALFF method proposed in this paper has an accuracy rate of $5.25 \%$ 242 higher than that in the literature [19]. In addition, the accuracy and AUC area of MCI vs NC,

243 MCI vs AD have been improved, because ALFF images can effectively reflect the activity of 244 neurons in each brain area of the subject, and are a good feature expression method. 245 Compared with the literature [19], fewer data sets were used in this paper and better 246 classification results are obtained, which proved that mALFF images have the positive role in 247 AD classification. 

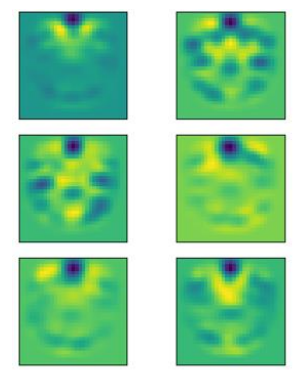

(a)
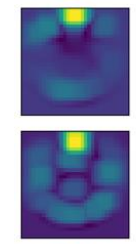

+u․
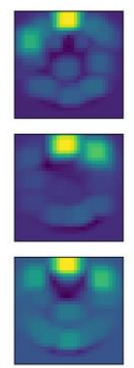

(c)
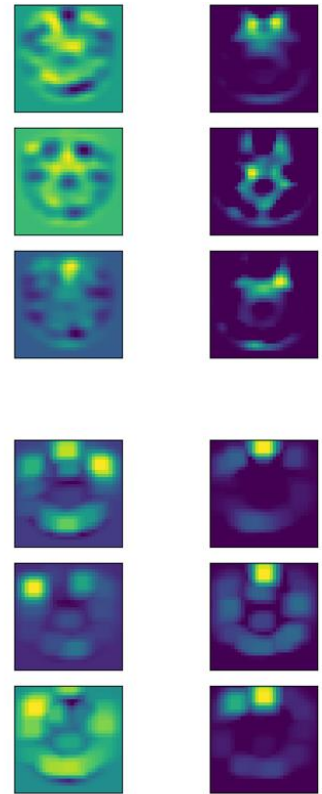
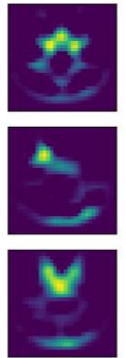

(b)
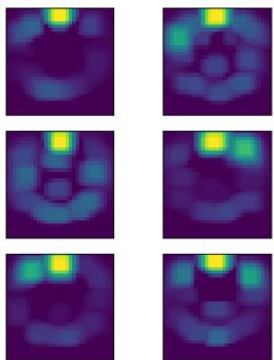

(d)
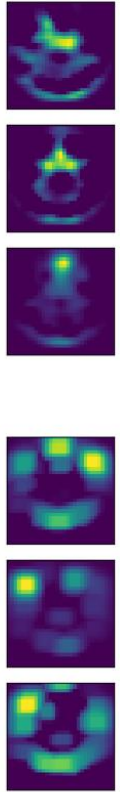

Fig.4 PCANet model outputted feature diagram Second, after the traditional 3DPCANet model is improved, the classification performance of
AD is significantly enhanced. For example, the accuracy, F1 value, specificity and other evaluation indicators of $\mathrm{NC}$ and $\mathrm{AD}, \mathrm{NC}$ and $\mathrm{EMCI}$ are improved, and the specificity of the latter isincreased by $12 \%$, AUC is increased by $11.66 \%$. Non-linear features of the image and the texture features of the image cannot extract by 3DPCANet. Fig4(a) presents the output feature map of the 3DPCANet convolutional layer. It can be seen from Figure (a) that the brain texture, and the structure of each brain area are not clear. In this paper. the convolved feature map pass in sequence through the ReLU layer, the max-pooling layer, and the combination layer (max-pooling and ReLU layer). The feature maps are shown in (b). (c), and (d) in Figure 4 respectively. In Figure 4 (b), the image sparsity increases after the feature map pass through the ReLU layer, highlighting the image features because the ReLU activation function changes the voxels with a voxel value less than 0 to 0 , and the voxel with a voxel value greater than 0 the voxel remains unchanged. After max-pooling, the feature map is compared with Figure 4 (a), texture features and brain structure of the image are revealed, because the max-pooling layer can learn the texture features of the image and reduce the redundant features of the image. Therefore, in this paper inserts the max-pooling layers and the ReLU layers after each convolutional layer in 3DPCANet, and the output feature map has a clear texture and large sparseness, thereby increasing the generalization ability of the model.

269 Thirdly, only AD and NC were classified in the literature [22], while other stages on AD 270 including SMC, EMCI and LMCI were also classified in this paper, which can assist medical personnel to diagnose patients in different stages of AD more accurately, and the results are of more practical significance. Compared with literature [23], [24], [25] and [26], the method proposed in this paper can more accurately diagnose patients at different stages of AD.

Fourth, the classification performance of SMC and LMCI, LMCI and AD also are improved, and MCI patients with weak pathological differences. The main reason is that since the 
maximum pooling layer is added behind the convolutional layer, redundant information in the feature map can be effectively reduced, over-fitting is prevented, and the activation function is increased. Therefore, the model can extract more nonlinear features, so that it is easy to distinguish the pathological differences of patients at different stages of development, and to get better classification performance.

As can be seen from the data in Table 3, because SMC and EMCI are the initial stage of AD, great differences on the brain structures between $\mathrm{AD}$ and SMC or EMCI exist. So, the classification results of SMC and $\mathrm{AD}$, and EMCI and $\mathrm{AD}$ are better, with the accuracy of 94.42\% and $88.42 \%$ respectively. $\mathrm{NC}$ and SMC, SMC and EMCI are the adjacent development stages, so small differences on brain structure exist, and it is difficult to distinguish. The accuracy of experimental results is $89.50 \%$ and $88.43 \%$, and the F1 value is $90.10 \%$ and $91.65 \%$, respectively. Both EMCI and LMCI belong to MCI stage, and it is difficult for medical personnel to distinguish patients in this stage. In this paper, the classification experiment of EMCI and LMCI also obtained good results, with F1 value of $87.44 \%$, AUC of $80.95 \%$ and accuracy of $84.21 \%$. The experimental results prove that the improved 3DPCANet model is more suitable for AD classification.

\section{Conclusions}

In this paper, an assisted diagnosis method of Alzheimer's disease based on deep learning is proposed. Firstly, mALFF images are obtained after fMRI data is pre-processed. Then Transformation images are inputted to an improved deep learning network in which the maxpooling layer and activation function layer are added behind the traditional 3DPCANet convolutional layer. This improvement can reduce the redundant information in the feature map, increase network depth and prevent over-fitting. Finally, SVM is used for classification of $\mathrm{AD}$ patients with different stages. The experimental results in the data set of AD and NC control group at different stages show that compared with the traditional 3DPCANet model, the classification results are notably improved. The highest accuracy on SMC vs. LMCI reaches $91.80 \%$, and that of LMCI vs. AD is $89.33 \%$. It can be seen from the results that $A D$ patients at different stages can be effectively classified using the proposed method in this paper. The research results can provide theoretical basis and guiding suggestions for the auxiliary diagnosis and treatment of AD.

Declarations

\section{Ethics approval and consent to participate}

309 In this paper, the dataset used is licensed by ADNI on September 24, 2019. ADNI gives the

310 permission statement: Congratulations. Your request for access to the Alzheimer's Disease

311 Neuroimaging Initiative (ADNI) Data has been approved. If you already had a LONI user 312 account your permissions have been updated to provide you access to ADNI data. If you did 313 not yet have an account, an account will be created for you and an e-mail with your account 314 information will be sent to you shortly.

\section{Consent for publication}

316 All authors agree to be published 


\section{Availability of data and material}

318 fMRI dataset used in this study comes from the Alzheimer's Disease Neuroimaging Initiative 319 (ADNI). The fMRI dataset includes 34 AD patients, 57 EMCI patients, 35 LMCI patients, 26 320 SMD patients, and 50 NC. Experimental data is obtained by sending an email to the ADNI 321 and signing the related agreement. Since in this laboratory the classification of Alzheimer's 322 disease is studied by the fusion of fMRI and sMRI image information, the subjects possessing

323 fMRI images and sMRI images are selected in the ADNI dataset. The link on ADNI dataset 324 is http://adni.loni.usc.edu/.

\section{Competing interests}

326 The authors declare that there is no conflict of interest regarding the publication of this manuscript.

\section{Funding}

329 This work is supported by Natural Science Foundation of China (No. 61671028), and Joint 330 Project of Beijing Natural Science Foundation and Beijing Municipal Education Commission 331 (No. KZ202110011015).

\section{Author Contributions}

$\mathrm{Yu}$ Wang is the corresponding author and the first author. Major contributions include fund, data set and experimental equipment acquisition, fusion algorithm ideas and program provider, supervision of experiments, and important modification of the paper.

336 Hongfei Jia, second author. Major contributions include experimental scheme and program design, model innovation, ALFF data preprocessing, and paper writer.

YifanDuan is the third author. Major contributions include REHO data preprocessing and paper modification.

340 Hongbing Xiao, the fourth author, Major contributions include fund acquisition and experimental background investigation.

343 Thanks to the Joint Project of Beijing Natural Science Foundation and Beijing Municipal Education Commission and Beijing Technology and Business University for their support

\section{References}

[1] Wang Q Y, Liang J H, Jia R X, et al. Prediction study of Alzheimer's disease in China from 2020 to 2050[J]. Alzheimer's disease and related diseases, 2019, 2(1): 289-298.

[2] Weiner M W, Veitch D P, Aisen P S, et al. Recent publications from the Alzheimer's Disease Neuroimaging Initiative: Reviewing progress toward improved AD clinical trials[J]. Alzheimer's \& Dementia, 2017, 13(4): 1-85. 
[4] Zeng H M, Han H B, Zhang Q F, et al. Application of modern neuroimaging technology in the diagnosis and study of Alzheimer's disease[J]. Neural Regeneration Research, 2021, 16(1):73-79.

355

356

357

358

359

360

361

362

363

364

365

366

367

368

369

370

371

372

373

374

375

376

377

378

379

380

381

382

383

384

385

386

387

[5] Yu S Y, Zhu W L, Guo P, et al. Clinical features and brain structural changes in magnetic resonance imaging in Alzheimer's disease patients with apathy[J]. Aging, 2020, 12(19):19083-19094.

[6] Golbabaei S, Vahid A, Hatami J, et al. Classification of Alzheimer's disease and mild cognitive impairment: Machine learning applied to rs-fMRI brain graphs[C] 2016 Iranian Conference on Biomedical Engineering and 2016 1st International Iranian Conference on Biomedical Engineering. Tehran, 24-25 Nov. 2016

[7] Fan Z, Xu F, Li C, et al. Application of KPCA and AdaBoost algorithm in classification of functional magnetic resonance imaging of Alzheimer's disease[J]. Neural Computing and Applications, 2020, 32(5): 5329-5338.

[8] Shi Y, Zeng W, Deng J, et al. The Identification of Alzheimer's Disease Using Functional Connectivity Between Activity Voxels in Resting-State fMRI Data[J]. IEEE Journal of Translational Engineering in Health and Medicine, 2020, 99: 1-1.

[9] Gui R, Chen T, Nie H. Classification of Task-State fMRI Data Based on Circle-EMD and Machine Learning[J]. Computational Intelligence and Neuroence, 2020, 2020(3):1-10.

[10] Zhou W, Wang Y, Xiao H B, et al. Auxiliary diagnosis of Alzheimer's disease based on KPCA algorithm[J]. Chinese Journal of Medical Physics, 2018, 035(004): 404-409.

[11] Li C S, Wang Y, Xiao H B, et al. Application of KPCA and Adaboost algorithm in the classification of functional magnetic resonance images of Alzheimer's disease[J]. Chinese Journal of Medical Physics, 2019, 36(007): 784-788.

[12] Asl E H, Keynton R, El-Baz A. Alzheimer's Disease Diagnostics by Adaptation of 3D Convolutional Network[C] 2016 IEEE International Conference on Image Processing - ICIP, Phoenix, AZ, 25-28 Sept. 2016.

[13] Lian C, Liu M, Zhang J, et al. Hierarchical Fully Convolutional Network for Joint Atrophy Localization and Alzheimer's Disease Diagnosis using Structural MRI[J]. IEEE Transactions on Pattern Analysis and Machine Intelligence, 2018: 880-893.

[14] Hosseini-Asl E, Gimel'Farb G, El-Baz A. Alzheimer's Disease Diagnostics by a Deeply Supervised Adaptable 3D Convolutional Network[J]. Front Bio, 2016, 23(3): 584-596.

[15] Billones C D, Demetria O J L D, Hostallero D E D, et al. DemNet: A Convolutional Neural Network for the detection of Alzheimer's Disease and Mild Cognitive Impairment[C] 2016 IEEE Region 10 Conference (TENCON), Singapore, 22-25 Nov. 2016.

[16] Jain R, Jain N, Aggarwal A, et al. Convolutional neural network based Alzheimer's disease classification from magnetic resonance brain images[J]. Cognitive Systems Research, 2019, 57: 147 159.

[17] Zhang F, Li Z, Zhang B, et al. Multi-modal Deep Learning Model for Auxiliary Diagnosis of Alzheimer's Disease[J]. Neurocomputing, 2019, 361(7): 185-199.

[18] Chan T H, Jia K, Gao S, et al. PCANet: A Simple Deep Learning Baseline for Image Classification?[J]. IEEE Transactions on Image Processing, 2015, 24(12): 5017-5032. 
[19] TONG LS, BIN X, SHENG LW, et al. Aided diagnosis of Alzheimer's disease based on 3D-PCANET [J]. Computer science, 2018, 45(6): 153-155

394 [20] Yan C G, Wang X D, Zuo X N, et al. DPABI: Data Processing \& Analysis for (Resting-State) Brain Imaging[J]. Neuroinformatics, 2016, 14(3): 339-351.

[21] Mu Y, Li Y, Zhang Q, et al. Amplitude of low-frequency fluctuations on Alzheimer's disease with depression: evidence from resting-state fMRI[J]. General Psychiatry, 2020, 33(4): e100147.

[22] Dai Z, Yan C, Wang Z, et al. Discriminative analysis of early Alzheimer's disease using multi-modal imaging and multi-level characterization with multi-classifier (M3) [J]. Neuroimage, 2012, 59(3): 2187-2195.

[23] Ashkan E, Dalboni D, Nagaraju, DB et al. Ensemble Classification of Alzheimer's Disease and Mild in Neuroence, 2017, :11-56

[24] Beheshti I, Maikusa N, Daneshmand M, et al. Classification of Alzheimer's Disease and Prediction of Mild Cognitive Impairment Conversion Using Histogram-Based Analysis of Patient-Specific Anatomical Brain Connectivity Networks[J]. Journal of Alzheimerl"s Disease, 2017, 60(1):295-304.

[25] Korolev S, Safiullin A, Belyaev M, et al. Residual and plain convolutional neural networks for 3D brain MRI classification[C]// IEEE International Symposium on Biomedical Imaging 2017. IEEE, 2017.

[26] Ys A, Zan W A, Pcb C, et al. Episodic Memory-related Imaging Features as Valuable Biomarkers for

Psychiatry: Cognitive Neuroscience and Neuroimaging, 2020. 


\section{Supplementary Files}

This is a list of supplementary files associated with this preprint. Click to download.

- datainBrief.zip 